\title{
Experiencia con el uso de teduglutide en pacientes pediátricos con fallo intestinal en un centro de Argentina
}

\author{
María Inés Martínez, Carolina Rumbo, Dolores García Hervás, Julio Trentadue, Gabriel \\ Gondolesi, Adriana Fernández
}

Programa de rehabilitación y trasplante intestinal, Hospital Universitario Fundación Favaloro, Ciudad Autónoma de Buenos Aires, Argentina.

Acta Gastroenterol Latinoam 2020;50(3):257-263

Recibido: 21/10/2019 / Aceptado: 17/07/2020 / Publicado online: 28/09/2020

\section{Resumen}

Introducción. El teduglutide es un análogo sintético del péptido simil glucagón tipo 2, aprobado recientemente en Europa y en los Estado Unidos para uso pediátrico, para promover la adaptación intestinal en casos de sindrome de intestino corto. La experiencia en niños es limitada. Objetivo. El objetivo de este trabajo es presentar la evolución de la primera serie pediátrica tratada con teduglutide en Argentina. Material $y$ métodos. Se realizó un estudio prospectivo sobre registros de pacientes menores de 18 años tratados en una unidad de rehabilitación y trasplante de intestino desde 2017. De 62 ninos con sindrome de intestino corto, 5 recibieron teduglutide 0,05 mg/kg/día subcutáneo. Se analizaron los diagnósticos, el tipo de anatomía y la evolución de los aportes de nutrición parenteral. Resultados. La etiología del sindrome de intestino corto fue igual a 3 malformaciones congénitas (2 atresias, 1 gastrosquisis); dos causas en niños mayores: 1 vólvulo y 1

Correspondencia: Adriana Fernández

Av. Belgrano 1782 (C.P.: 1093), Programa de rehabilitación y trasplante intestinal, Fundación Favaloro, Hospital Universitario.

Ciudad Autónoma de Buenos Aires, Argentina

Tel.: (+54)221534416

Correo electrónico: adrianafernandezlp@gmail.com trauma abdominal. La anatomía de los pacientes era tres tipo $2 A$, uno tipo $2 B$ y uno tipo $3 A$. La longitud del intestino remanente (media y $D S$ ) fue de $25,7 \pm 19 \mathrm{~cm}, 1$ con válvula ileocecal y colon, 4 hemicolon izquierdo. La edad al inicio del aporte de nutrición parenteral fue 6,2 $\pm 0,4$ años, el tiempo de aporte de nutrición parenteral previo fue de 7,1 4 años. El tiempo de tratamiento con teduglutide fue de 52,2 \pm 39 semanas. El aporte de nutrición parenteral se redujo en $56 \pm$ $48 \%$, en todos los pacientes, pudiendo suspenderse en 2 (a las semanas $29^{\circ}$ y $24^{\circ}$ del tratamiento). No se registró deterioro del z score de IMCledad (0,16 inicial $\pm 0,3$ vs. 0,14 $\pm 1,02$ al corte), ni de la tallaledad $(-2,01 \pm 1,5$ vs. $-1,76 \pm 1,42)$. Conclusión. Se llegó a la conclusión de que el uso de teduglutide como alternativa terapéutica en el sindrome de intestino corto en pediatría resultó efectiva y segura en este grupo de pacientes, permitiendo restaurar la suficiencia intestinal o reduciendo los aportes de nutrición parenteral.

Palabras claves. Sindrome de intestino corto, nutrición parenteral, fallo intestinal, teduglutide, pediatría.

\section{Experience with the use of teduglutide in paediatric patients with intestinal failure in a center in Argentina}

\section{Summary}

Introduction. Teduglutide is a synthetic analogue of the glucagon-like peptide type 2, recently approved in Europe and in the United States for paediatric use, to promote intestinal adaptation in short bowel syndrome cases. The experience in 
children is limited. Objective. The aim of this work is to present the evolution of the first paediatric series treated with teduglutide in Argentina. Material and methods. A prospective study was realized on patient records under 18 years treated in a rehabilitation and intestine transplant unit since 2017. Of 62 children with short bowel syndrome, 5 received teduglutide $0.05 \mathrm{mg} / \mathrm{kg} / \mathrm{subcutaneous} \mathrm{day.} \mathrm{Diagnostics,} \mathrm{type}$ of anatomy and evolution of parenteral nutrition requirements were realized. Results. Etiology of short bowel syndrome: 3 congenital malformations: 2 atresias, 1 gastroschisis, two causes in older children: 1 volvulus and 1 abdominal trauma. Anatomy. Three patients type $2 A$, one type $2 B$ and one type $3 A$, the length of the remaining intestine (mean and DS) $25.7 \pm 19 \mathrm{~cm}, 1$ with ileocecal valve and colon, 4 left hemicolon. The age at the beginning of parenteral nutrition was $6.2 \pm 0.4$ years, time on previous parenteral nutrition was $7.1 \pm 4$ years. The treatment time with teduglutide was $52.2 \pm 39$ weeks. The parenteral nutrition requirements were reduced by $56 \pm 48 \%$, in all patients, and could be suspended in 2 (at the 29th and 24th weeks of treatment). There was no deterioration of the $z$ score of BMIlage (initial $0.16 \pm 0.3 \mathrm{vs.}$ $0.14 \pm 1.02)$, nor of the heightlage $(-2.01 \pm 1.5$ vs. $-1.76 \pm$ 1.42). Conclusion. It was concluded that the use of teduglutide as a therapeutic alternative in the short bowel syndrome in paediatrics was effective and safe in this group of patients, allowing the restoration of intestinal sufficiency or reducing the requirements of parenteral nutrition.

Key words. Short bowel syndrome, parenteral nutrition, intestinal failure, teduglutide, paediatric.

\section{Introducción}

El tratamiento de los pacientes pediátricos con falla intestinal crónica (FIC) secundaria a síndrome de intestino corto (SIC) ha evolucionado favorablemente en los años recientes con el establecimiento de equipos interdisciplinarios especializados en su tratamiento, los avances en las formulaciones de nutrición parenteral (NP), el desarrollo de técnicas quirúrgicas y los cuidados perioperatorios. ${ }^{1,2}$

Con un manejo adecuado, la mayor parte de los pacientes pediátricos con SIC va a alcanzar la adaptación intestinal y podrá ser destetado de la NP. Este proceso de adaptación intestinal ha sido descripto, ${ }^{3}$ pero los mecanismos que lo determinan fueron pobremente entendidos. Se han involucrado distintos aspectos, factores hormonales tales como: hormona de crecimiento, péptido símil glucagón 1 (GLP-1), péptido símil glucagón tipo 2 (GLP-2) y factores liminales (ácidos grasos de cadena larga, proteínas, glutamina, prebióticos), entre otros. ${ }^{4,5}$

El GLP-2 sería un regulador clave en el proceso de adaptación intestinal. ${ }^{6,7} \mathrm{El}$ teduglutide, un análogo sintético del GLP-2 con vida media más prolongada, se ha desarrollado recientemente como un tratamiento específico para promover la adaptación intestinal en los pacientes con el SIC..$^{8,9}$

Los reportes del uso del teduglutide en adultos han demostrado seguridad y efectividad con su uso prolongado. ${ }^{10,11}$ La droga fue aprobada para el uso en pacientes de población adulta con SIC en Estados Unidos por la FDA y en la Unión Europea (EMA) en el año 2012. La experiencia clínica con el uso de teduglutide en edad pediátrica es limitada; en Europa se autorizó en niños de 1 a 18 años en 2016 y en Estados Unidos en 2019. Argentina es el primer país en Latinoamérica con experiencia en el uso de teduglutide en pacientes adultos y pediátricos. Como es una droga que aún no ha sido aprobada por ANMAT (Administración Nacional de Medicamentos, Alimentos y Tecnología Médica), su utilización se realiza bajo el Régimen de Acceso de Excepción a Medicamentos, y con autorización del Comité de Ética Asistencial de la Institución.

El objetivo de esta presentación es describir nuestra experiencia inicial con el uso del teduglutide en pacientes pediátricos con el SIC.

\section{Material y métodos}

Se realizó un estudio prospectivo descriptivo sobre registros de pacientes $<18$ años tratados en la unidad de rehabilitación y trasplante de intestino del Hospital Universitario Fundación Favaloro de Argentina, desde enero de 2017. Sobre 62 niños con SIC en seguimiento desde el año 2006: 22 recibieron Trasplante de Intestino, 14 fallecieron, 12 se adaptaron, 9 continúan en NP y a 5 pacientes dependientes de NP se les indicó teduglutide en una dosis diaria de $0,05 \mathrm{mg} / \mathrm{kg} /$ día por vía subcutánea. Se analizaron los diagnósticos, el tipo de anatomía remanente, el tiempo de evolución previa de la FI, la evolución de los aportes parenterales (NP) entre el inicio del teduglutide y el corte, expresados como número de infusiones de nutrición parenteral por semana y porcentaje del gasto metabólico basal (GMB) estimado con la ecuación de Schofiel según edad y sexo, cubierto como $\mathrm{kcal}$ no proteicas (aportadas como glucosa y lípidos) en la NP (Cf. Goulet, 2018). ${ }^{12}$

También se describió la evolución del estado nutricional expresado como z score del índice de masa corporal (IMC) y la talla para la edad y la presencia de eventos adversos. 
Se diseñó un protocolo de seguimiento que incluía un monitoreo inicial y periódico clínico, antropométrico, de aportes nutricionales parenterales, orales y enterales, de registro de catarsis y diuresis, laboratorios y estudios de imágenes para valorar la seguridad y eficacia del tratamiento. Antes de iniciar el tratamiento se realizaron estudios complementarios basales que incluían determinaciones de laboratorio: un hemograma, un hepatograma, el estado ácido base venoso, la función renal y hepática, la albúmina, la glucemia, la trigliceridemia, la amilasemia, el ionograma plasmático y en la orina, el estado nutricional del hierro, la determinación de $\mathrm{OH}$ vitamina $\mathrm{D}$, el ácido fólico, la vitamina B12 y una ecografía abdominal. Se les realizó una videoendoscopía digestiva baja (VEDB) para la detección de pólipos a los pacientes mayores de 12 años y determinación por anticuerpos de sangre oculta en materia fecal a los pacientes menores de 12 años, con posterior realización de una VEDB si ésta resultaba positiva. Se determinó la citrulina plasmática por cromatografía líquida de alta performance (HPLC), según la disponibilidad al inicio del tratamiento y luego de 6 meses a 1 año de iniciado.

Los valores son expresados en números totales, resumidos en medias $(\mathrm{M})$ y desvíos estándar $( \pm)$ y las diferencias analizadas con el test de t para muestras apareadas con el programa SPSS Stat 24.

El presente estudio se realizó bajo la aprobación del Comité de Ética de la Fundación Favaloro (DDI (1478) 1219) y todos los padres y pacientes que participaron firmaron un consentimiento o un asentimiento informado para su inclusión.

\section{Resultados}

La indicación del tratamiento con teduglutide se realizó en los pacientes que habían alcanzado una meseta en el proceso de adaptación intestinal, luego de haber implementado otras estrategias de rehabilitación médicas, quirúrgicas y nutricionales. Los pacientes se consideraban dentro de una meseta de su proceso de adaptación intestinal cuando habían trascurrido al menos 2 años desde la resección quirúrgica y, en al menos el último año, no se había logrado reducir el aporte parenteral (en \% del aporte calórico respecto al GMB o en el $\mathrm{N}^{\circ}$ de infusiones de NP semanales). Esta evaluación se realizó tomando en cuenta los parámetros antropométricos, los aportes enterales, las características de las deposiciones y los valores de los laboratorios (electrolitos, minerales, EAB, urea y albúmina).

El manejo médico incluyó antisecretores solo durante los primeros 6 a 12 meses después de la resección intestinal y colestiramina como quelante de sales biliares duran- te los primeros meses luego de la anastomosis a colon. Se utilizó la loperamida como antidiarreico $(0,5$ a $1 \mathrm{mg} / \mathrm{k} / \mathrm{d}$ hasta $16 \mathrm{mg} / \mathrm{d}$ ) en 3 a 4 dosis diarias y metronidazol ante la sospecha clínica de sobredesarrollo bacteriano $(25-30 \mathrm{mg} / \mathrm{k} / \mathrm{d})$, con frecuencia de tratamiento ajustada para cada paciente.

La tabla 1 muestra las características de los pacientes al momento del inicio del tratamiento en cuanto a las causas del SIC, la edad al momento de la resección intestinal y al inicio del tratamiento, la anatomía remanente y el dosaje de citrulina. El tipo de SIC se clasificó en tipo 1 (yeyunostomía terminal), tipo 2 (yeyuno colon anastomosis) y tipo 3 (yeyuno-íleo anastomosis con conservación de VIC y todo el colon). Además, según la longitud de intestino delgado remanente se subclasificaron en $\mathrm{A}(<$ de $40 \mathrm{~cm})$ y B $(>40 \mathrm{~cm})$.

El SIC tuvo inicio neonatal por malformaciones congénitas en 3 pacientes ( 2 atresias intestinales y 1 con gastrosquisis y atresia) y en dos casos en edad pediátrica, uno por vólvulo y otro por traumatismo con lesión de la arteria mesentérica superior. La determinación de citrulina plasmática al inicio se realizó en 3 pacientes con valores entre 7 y $15 \mathrm{Umol} / \mathrm{L}$ y mostró mejoría en los 2 pacientes en los que se repitió: en el paciente $n^{\circ} 2$ en la semana $41,20,3 \mathrm{Umol} / \mathrm{L}$ y en el paciente $\mathrm{n}^{\circ} 3$ en la semana 23, 21,3 Umol/L.

Los pacientes habían recibido NP previamente durante un rango de 2,7 a 12,7 años, con aportes de 79 al $100 \%$ del GMB estimado y entre 6 y 7 infusiones de NP por semana. El tiempo de tratamiento con el teduglutide fue de X 52,2 \pm 39 semanas. En ningún paciente se suspendió.

La tabla 2 muestra la evolución de los aportes parenterales y el porcentaje de disminución de los mismos al momento del corte (entre 9 y 101 semanas de tratamiento) (Figuras 1 y 2). Dos pacientes pudieron ser destetados de la NP luego de 29 (paciente $\mathrm{n}^{\circ} 1$ ) y 24 semanas de tratamiento (paciente $n^{\circ} 2$ ) y ambos permanecen sin NP al corte. En los otros 3 pacientes se redujeron tanto los aportes parenterales como el número de infusiones semanales. La tabla 3 muestra la evolución del estado nutricional. Se observa que no hubo deterioro a pesar de la reducción de los aportes parenterales. Los eventos adversos más frecuentemente reportados fueron dolor abdominal leve $y$ autolimitado en 3 pacientes y hematomas en los sitios de inyección en 2 . Otros eventos reportados fueron faringitis e infección de la vía aérea superior. El paciente $n^{\circ} 1$ presentó determinación de SOMF positiva, sin anemia, solicitada según el protocolo de seguimiento al año de tratamiento y se le realizó una videoendoscopia digestiva alta y baja en las que no se encontraron lesiones. 
Tabla 1. Antecedentes de los pacientes al inicio

\begin{tabular}{|c|c|c|c|c|c|}
\hline $\begin{array}{l}\text { Paciente } \\
\text { sexo }\end{array}$ & $\begin{array}{l}\text { Diagnóstico } \\
\text { de base }\end{array}$ & $\begin{array}{l}\text { Edad inicio } \\
\text { Fl (años) }\end{array}$ & $\begin{array}{l}\text { Tiempo de Fl al inicio } \\
\text { Teduglutide (años) }\end{array}$ & $\begin{array}{l}\text { Anatomía } \\
\text { remanente }\end{array}$ & $\begin{array}{l}\text { Citrulina } \\
\text { inicial }\end{array}$ \\
\hline $\begin{array}{l}\mathrm{N}^{0} 1 \\
\text { Masculino }\end{array}$ & $\begin{array}{l}\text { Atresia IIIA } \\
\text { Ano imperforado }\end{array}$ & 0 & 6 & $\begin{array}{l}\text { Tipo } 3 \mathrm{~A} \\
20 \mathrm{~cm} \text { yeyuno }+3 \mathrm{~cm} \text { íleon } \\
\text { VIC + colon completo }\end{array}$ & $\begin{array}{l}\text { No } \\
\text { realizada }\end{array}$ \\
\hline $\begin{array}{l}\mathrm{N}^{0} 2 \\
\text { Masculino }\end{array}$ & Vólvulo & 6,5 & 11,4 & $\begin{array}{l}\text { Tipo } 2 \mathrm{~A} \\
30 \mathrm{~cm} \text { yeyuno } \\
\text { Hemicolon izquierdo }\end{array}$ & 7,0 \\
\hline $\begin{array}{l}\mathrm{N}^{0} 3 \\
\text { Masculino }\end{array}$ & $\begin{array}{l}\text { Gastrosquisis } \\
\text { y atresia }\end{array}$ & 0 & 12,7 & $\begin{array}{l}\text { Tipo } 2 \mathrm{~A} \\
8 \mathrm{~cm} \text { yeyuno } \\
\text { Hemicolon izquierdo }\end{array}$ & 15,7 \\
\hline $\begin{array}{l}\mathrm{N}^{0} 4 \\
\text { Femenino }\end{array}$ & $\begin{array}{l}\text { Trauma } \\
\text { abdominal }\end{array}$ & 10,1 & 2,7 & $\begin{array}{l}\text { Tipo } 2 \mathrm{~A} \\
15 \mathrm{~cm} \text { yeyuno } \\
\text { Hemicolon izquierdo }\end{array}$ & 8,2 \\
\hline $\begin{array}{l}\text { No } 5 \\
\text { Masculino }\end{array}$ & Atresia & 0 & 7,0 & $\begin{array}{l}\text { Tipo 2B } \\
50 \mathrm{~cm} \text { yeyuno } \\
\text { Hemicolon izquierdo }\end{array}$ & $\begin{array}{l}\text { No } \\
\text { realizada }\end{array}$ \\
\hline & & $\begin{array}{l}\text { M 3,4 } \\
\pm 5,01\end{array}$ & $\begin{array}{l}\text { M 7,9 } \\
\pm 4,08\end{array}$ & $\begin{array}{l}\text { M LIR 25,7 } \\
\pm 19 \mathrm{~cm}\end{array}$ & \\
\hline
\end{tabular}

Tabla 2. Resultados del tratamiento en relación a la dependencia de nutrición parenteral

\begin{tabular}{|c|c|c|c|c|c|c|}
\hline Paciente & $\begin{array}{l}\text { Semanas de } \\
\text { tratamiento }\end{array}$ & $\begin{array}{l}\text { Infusiones } \\
\text { por semana } \\
\text { al inicio }\end{array}$ & $\begin{array}{l}\text { Infusiones } \\
\text { por semana } \\
\text { al corte }\end{array}$ & $\begin{array}{l}\text { Aporte } \\
\text { inicial (\%) }\end{array}$ & $\begin{array}{l}\text { Aporte } \\
\text { final (\%) }\end{array}$ & $\begin{array}{l}\% \text { de reducción } \\
\text { del aporte calórico }\end{array}$ \\
\hline No 1 & 101 & 6 & 0 & 80 & 0 & 100 \\
\hline$N^{0} 2$ & 84 & 6 & 0 & 98 & 0 & 100 \\
\hline$N^{0} 3$ & 41 & 7 & 5 & 83 & 62 & 25 \\
\hline$N^{0} 4$ & 26 & 6 & 5 & 79 & 44 & 45 \\
\hline \multirow[t]{3}{*}{ № 5} & 9 & 6 & 6 & 101 & 87 & 14 \\
\hline & M 52,9 & M 6,2 a & $M 3,2^{a}$ & $M 88,2^{b}$ & $M 38,6^{b}$ & M 56,8 \\
\hline & \pm 39 & $\pm 0,44$ & $\pm 2,9$ & $\pm 10,4$ & $\pm 38,4$ & $\pm 40,9$ \\
\hline
\end{tabular}

$p=0,04$ 
Figura 1. Número de infusiones semanales de NP por paciente al momento del corte

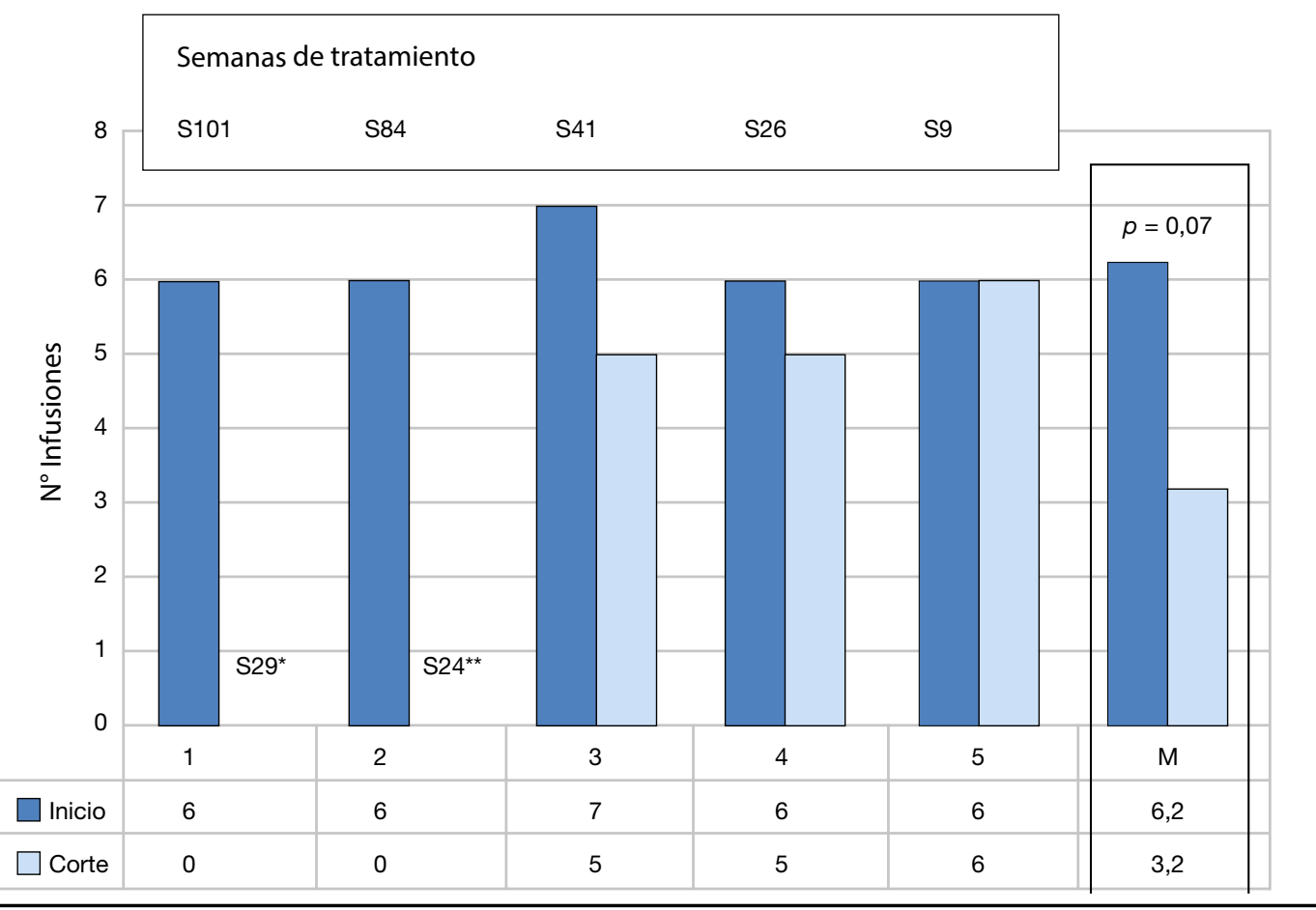

Se muestra la evolución del número de infusiones expresado por semanas (S) de tratamiento en la parte superior. Se mencionan la semana $29^{\star}\left(\right.$ S29) y la semana $24^{\star *}(\mathrm{~S} 24)$ en las que los pacientes 1 y 2 suspendieron la NP.

Figura 2. Porcentaje del GMB aportado como Kcal no proteicas en NP al momento del corte

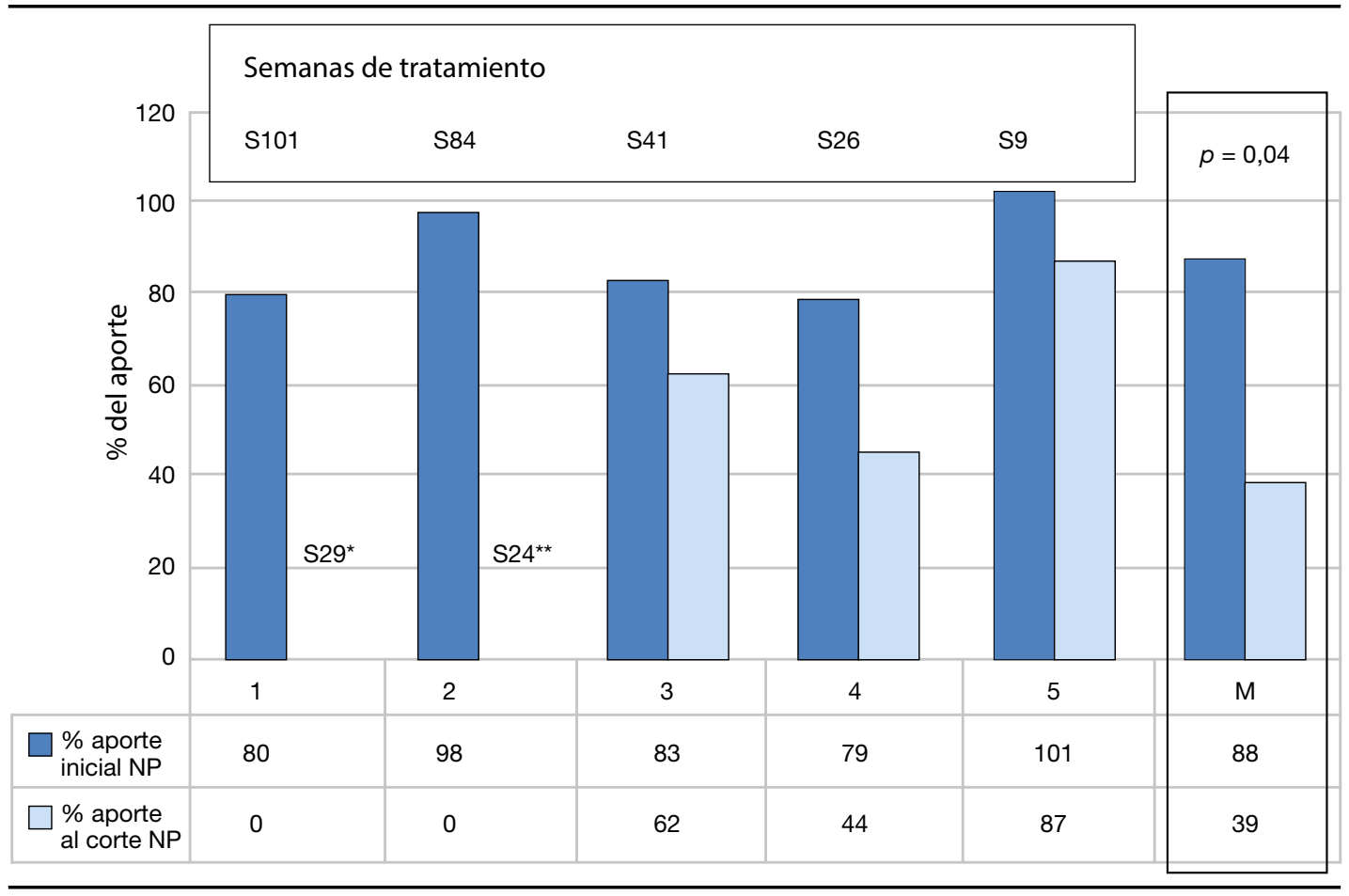

Se muestra la evolución del aporte calórico expresado por semanas (S) de tratamiento en la parte superior. Se mencionan la semana $29^{\star}\left(\right.$ S29) y la semana $24^{\star *}$ (S24) en las que los pacientes 1 y 2 suspendieron la NP. 
Tabla 3. Evolución de los parámetros antropométricos y efectos adversos

\begin{tabular}{|c|c|c|c|c|c|}
\hline Paciente & $\begin{array}{l}\text { Z score } \\
\text { IMC/edad } \\
\text { inicial }\end{array}$ & $\begin{array}{l}\text { Z score } \\
\text { IMC/edad } \\
\text { al corte }\end{array}$ & $\begin{array}{l}\text { Z score } \\
\text { Talla/edad } \\
\text { inicial }\end{array}$ & $\begin{array}{l}\text { Z score } \\
\text { Talla/edad } \\
\text { al corte }\end{array}$ & $\begin{array}{l}\text { Efectos } \\
\text { adversos }\end{array}$ \\
\hline № 1 & 1,32 & 0,97 & $-3,66$ & $-2,61$ & $\begin{array}{l}\text { Infección respiratoria alta. } \\
\text { Hematomas en sitio de inyección. }\end{array}$ \\
\hline № 2 & $-0,68$ & $-1,3$ & $-2,01$ & $-2,02$ & Dolor abdominal. \\
\hline № 3 & $-0,27$ & $-0,59$ & $-1,70$ & $-1,61$ & $\begin{array}{l}\text { Faringitis epigastralgia. } \\
\text { Hematomas en sitio de inyección. }\end{array}$ \\
\hline NN 4 & 0,28 & 0,80 & 0,30 & 0,29 & Dolor abdominal. \\
\hline \multirow[t]{3}{*}{ № 5} & 0,18 & 0,84 & $-2,97$ & $-2,84$ & Infección respiratoria alta. \\
\hline & M 0,16 & M 0,14 & $M-2,1$ & $M-1,76$ & \\
\hline & $\pm 0,38$ & $\pm 1,02$ & $\pm 1,5$ & $\pm 1,42$ & \\
\hline
\end{tabular}

\section{Discusión}

Los pacientes descritos en este reporte tenían falla intestinal grave, 3 con anatomía tipo $2 \mathrm{~A}$, uno tipo $2 \mathrm{~B}$ y uno $3 \mathrm{~B}$, con dependencia alta de NP por un tiempo prolongado, mínimo de 2.7 años, previo al inicio del tratamiento con el teduglutide. La anatomía tipo 1 no fue un criterio de exclusión. Todos los pacientes en seguimiento con NP en el momento de este análisis estaban reconstruidos al iniciar el estudio.

Se les indicó el tratamiento cuando se consideraron agotados los tratamientos médicos, nutricionales y quirúrgicos implementados por el equipo multidisciplinario para lograr la adaptación intestinal.

El receptor del GLP-2 se expresa a lo largo del intestino delgado, en todos los grupos etarios, incluyendo prematuros. ${ }^{12}$ Actúa aumentando la proliferación epitelial en las criptas, reduciendo la apoptosis epitelial, aumentando el flujo sanguíneo intestinal, enlenteciendo la motilidad intestinal, con un efecto trófico para la mucosa del intestino delgado. El GLP-2 no actúa como señal directamente sobre las células mucosas. Sus efectos tróficos son mediados por los segundos efectores, probablemente por la producción de GF-1 por los miofibroblastos. Algunos de estos efectos podrían estar mediados, al menos en parte, por la estimulación de vías neuronales entéricas por el GLP-2. ${ }^{13,14}$

El análogo del GLP-2 emerge como una nueva alternativa de tratamiento para los pacientes pediátricos, y su eficacia y seguridad ha sido demostrada en estudios de corto plazo. ${ }^{15}$

Se ha publicado recientemente un estudio de seguimiento de pacientes pediátricos observados hasta la semana 24 de tratamiento. ${ }^{16}$ Sobre 59 pacientes incluidos en el estudio, el $54 \%$ de los pacientes logró reducir más del $20 \%$ del aporte de NP con una dosis de teduglutide de $0,025 \mathrm{mg} / \mathrm{kg} / \mathrm{d}$, el $69 \%$ con una dosis de $0,05 \mathrm{mg} / \mathrm{kg} / \mathrm{d}$ y $11 \%$ con cuidados estándar; 5 pacientes lograron suspender la NP durante el período observado.

Es importante tener en cuenta que debido a que el GLP-2 tiene un efecto trófico en la mucosa intestinal, podría determinar un mayor riesgo de inducir el crecimiento de pólipos localizados, o en casos ominosos la malignización de los mismos. ${ }^{17} \mathrm{El}$ test de SOMF fue realizado como un requisito para iniciar el tratamiento en niños menores de 12 años. En el paciente $n^{\circ} 1$ fue negativo al inicio y en el paciente $n^{\circ} 5$ fue positivo. Se realizó una $\mathrm{VEDB}$ en este paciente y en los tres pacientes mayores de 12 años al inicio (pacientes $n^{\circ} 2,3$ y 4) sin hallazgo de pólipos.

Los efectos adversos reportados en estudios en población adulta incluyen: complicaciones locales en el sitio de inyección (dolor persistente, induración, hematomas, alergias a la droga o al solvente); alteraciones del tubo digestivo (vómitos, náuseas, distensión abdominal, ausencia de deposiciones, dolor abdominal, oclusión intestinal, edema o disfunción de la ostomía); infecciones respirato- 
rias; alteración del hepatograma, descenso de plaquetas, esplenomegalia; edemas principalmente de miembros inferiores; descenso de peso; dolor musculo esquelético, calambres y astenia. En esta serie los eventos adversos encontrados fueron leves y autolimitados. ${ }^{18,19}$

La experiencia con esta serie es de pocos pacientes y la duración del tratamiento es relativamente corta. Se incluyeron aquellos pacientes en los que no se pudo lograr adaptación intestinal ni progreso en la disminución de los aportes de NP por un período prolongado. El criterio de selección fue el mencionado a lo que debimos sumar la aceptación del tratamiento por su seguro social.

Estos primeros resultados sugieren que el teduglutide es una terapia apropiada para reducir o aun suspender el aporte parenteral en pacientes con FIC por SIC.

El desafío entre los equipos de expertos que asisten a estos niños es identificar los candidatos que podrían beneficiarse con este tratamiento y reconocer el momento oportuno de su indicación en la historia natural de la enfermedad y del proceso de la rehabilitación intestinal.

Poniendo en perspectiva la historia de 13 años de nuestro programa, que incluye a la serie más grande de trasplante de intestino pediátrico en Latinoamérica, el teduglutide ha emergido como una opción de tratamiento para ofrecerle a los pacientes una vez que fueron implementaros los otros componentes de la rehabilitación intestinal sin alcanzarse la adaptación. Los resultados favorables obtenidos hasta el momento alientan el uso del teduglutide en pacientes con fallo intestinal por SIC, siendo necesario conocer los efectos de la droga a largo plazo.

\section{Referencias}

1. Goulet O, Nader EA, Pigneur B, Lambe C. Short Bowel Syndrome as the Leading Cause of Intestinal Failure in Early Life: Some Insights into the Management. Pediatr Gastroenterol Hepatol Nutr 2019; 22 (4): 303-329.

2. Jaksic T, Modi B. Short bowel syndrome. Semin Pediatr Surg 2018; 27 (4): 207-208.

3. Buchman AL. Intestinal Failure and Rehabilitation. Gastroenterol Clin North Am 2018; 47 (2): 327-340.

4. Courtney CM, Onufer EJ, Seiler KM, Warner BW. An anatomic approach to understanding mechanisms of intestinal adaptation. Semin Pediatr Surg 2018; 27 (4): 229-236.

5. Billiauws L, Thomas M, Le Beyec-Le Bihan J, Joly F. Intestinal adaptation in short bowel syndrome. What is new? Nutr Hosp 2018; 35 (3): 731-737.

6. Jeppesen PB, Hartmann B, Thulesen J, Graff J, Lohmann J, Hansen BS, Tofteng F, Poulsen SS, Madsen JL, Holst JJ, Mortensen PB. Glucagon-like peptide 2 improves nutrient absorption and nutritional status in short-bowel patients with no colon. Gastroenterology 2001; 120 (4): 806-815.
7. Mutanen A, Pakarinen MP. Serum fasting GLP-1 and GLP-2 associate with intestinal adaptation in pediatric onset intestinal failure. Clin Nutr 2017; 36 (5): 1349-1354.

8. Schwarts LK, O’Keefe S, Fujioka K, Gabe SM, Lamprecht G, Pape UF, Li B, Youssef NN, Jeppesen PB. Long-Term Teduglutide for the Treatment of Patients With Intestinal Failure Associated With Short Bowel Syndrome. Clinical and Transl Gastroenterology 2016; 7 (2): e142.

9. Kim ES, Keam SJ. Teduglutide: A Review in Short Bowel Syndrome. Drugs 2017; 77 (3): 345-352.

10. Seidner DL, Fujioka K, Boullata JI, Iyer K, Lee HM, Ziegler TR. Reduction of Parenteral Nutrition and Hydration Support and Safety With Long-Term Teduglutide Treatment in Patients With Short Bowel Syndrome-Associated Intestinal Failure: STEPS-3 Study. Nutr Clin Pract 2018; 33 (4): 520-527.

11. Iyer KR, Kunecki M, Boullata JI, Fujioka K, Joly F, Gabe S, Pape UF, Schneider SM, Virgili Casas MN, Ziegler TR, Li B, Youssef NN, Jeppesen PB. Independence From Parenteral Nutrition and Intravenous Fluid Support During Treatment With Teduglutide Among Patients With Intestinal Failure Associated With Short Bowel Syndrome. JPEN J Parenter Enteral Nutr 2017; 41 (6): 946-951.

12. Abi Nader E, Lambe C, Talbotec C, Dong L, Pigneur B, Goulet O. A New Concept to Achieve Optimal Weight Gain in Malnourished Infants on Total Parenteral Nutrition. JPEN J Parenter Enteral Nutr 2018; 42 (1): 78-86.

13. Sigalet DL, Martin G, Meddings J, Hartman B, Holst JJ. GLP-2 levels in infants with intestinal dysfunction. Pediatr Res 2004; 56 (3): 371-376.

14. Sigalet DL. Advances in glucagon like peptide-2 therapy. physiology, current indications and future directions. Seminars in Pediatric Surgery 2018; 27 (4): 237-241.

15. Sigalet DL, Brindle ME, Boctor D, Dicken B, Lam V, Lu LS, de Heuvel E, Hartmann B, Holst JJ. A safety and pharmacokinetic dosing study of glucagon-like peptide 2 in infants with intestinal failure. J Ped Surg 2017; 52 (5): 749-754.

16. Carter BA, Cohran VC, Cole CR, Corkins MR, Dimmitt RA, Duggan C, Hill S, Horslen S, Lim JD, Mercer DF, Merritt RJ, Nichol PF, Sigurdsson L, Teitelbaum DH, Thompson J, Vanderpool C, Vaughan JF, Li B, Youssef NN, Venick RS, Kocoshis SA. Outcomes from a 12-week, open-label, multicenter clinical trial of Teduglutide in pediatric short bowel syndrome. J Pediatrics 2017; 181: 102-111.

17. Kocoshis SA, Merritt RJ, Hill S, Protheroe S, Carter BA, Horslen S, Hu S, Kaufman SS, Mercer DF, Pakarinen MP, Venick RS, Wales PW, Grimm AA. Safety and Efficacy of Teduglutide in Pediatric Patients With Intestinal Failure due to Short Bowel Syndrome: A 24-Week, Phase III Study. JPEN J Parenter Enteral Nutr 2020; 44 (4): 621-631. Disponible en doi: 10.1002/ jpen.1690.

18. Iakoubov R, Lauffer LM, Trivedi S, Kim YJ. Carcinogenic effects of exogenous and endogenous glucagon-like peptide-2 in azoxymethane-trested mice. Endocrinilogy 2009; 150 (9): 4033-4043.

19. O'Keefe SJD, Jeppesen PB, Gilroy R, Pertkiewicz M, Allard JP, Messing B. Safety and efficacy of teduglutide after 52 weeks of treatment in patients with short bowel intestinal failure. Clin Gastroenterol Hepatol 2013; 11 (7): 815-823.

20. Kim ES, Keam SJ. Teduglutide: A Review in Short Bowel Syndrome. Drugs. 2017; 77 (3): 345-352. 